\title{
Pauli, Schrödinger und der Streit um die Deutung der Quantentheorie
}

Von Karl von Meyenn

\section{Welle, Teilchen und andere begriffliche Schwierigkeiten \\ in der älteren Quantentheorie}

Obwohl in der Einsteinschen Lichtquantenhypothese die Problematik des Welle-Teilchen-Dualismus beim Licht schon frühzeitig sichtbar geworden war, kam ihre Aufklärung nur langsam voran ${ }^{1}$. «Der Schwerpunkt der ganzen Frage scheint mir darin zu liegen», resümierte der damals noch als Privatdozent an der Universität Zürich wirkende Einstein: «Lassen sich die Energiequanten einerseits und das Huygensche Prinzip andererseits vereinigen? Der Schein ist dagegen, aber der Herrgott hat - wie es scheint - doch den Rank gefunden.» ${ }^{2}$ Die von Einstein sogenannte «h-Krankheit» machte sich aber auf anderen Gebieten bemerkbar und ließ schon damals erste Zweifel an der allgemeinen Gültigkeit der Mechanik aufkommen ${ }^{3}$.

Noch viel deutlicher zeichnete sich der Gegensatz zwischen der klassischkontinuierlichen und der quantentheoretisch-statistischen Beschreibung der Strahlungsvorgänge in Einsteins statistischer Herleitung des Planckschen Strahlungsgesetzes aus dem Jahre 1916 ab. Einstein hatte zu diesem Zweck ein Gesetz für das Auftreten der spontanen Emissionsprozesse formuliert, das dem statistischen Gesetz des radioaktiven Zerfalls nachgebildet war. Dieses Gesetz sagte jedoch nur etwas über das mittlere Verhalten vieler Atome aus. Insbesondere war in dieser Formulierung nichts über den Zeitpunkt des Übergangs eines einzelnen angeregten Atoms vorausgesetzt. Sofern es sich also nicht nur um eine provisorische Unvollkommenheit der Theorie handelte, mußte man diese Tatsache als ein Anzeichen für ein grundsätzliches Versagen der kausalen Naturbeschreibung auslegen ${ }^{4}$.

Die paradoxen Folgerungen, zu denen die Doppelnatur des Lichtes führte, wurden Anfang der 20 er Jahre von Einstein in mehreren Gedankenexperimenten eingehend analysiert. «Es ist gut», klagte er seinem Freunde Paul Ehrenfest 1921, «daß ich soviel Ablenkungen habe, denn sonst hätte mich das Quantenproblem längst ins Irrenhaus gebracht. Daß Licht, was nach diametral verschiedenen Richtungen emittiert ist, interferieren kann, 
soll einwandfrei nachgewiesen sein. Wie soll das mit der energetischen Gerichtetheit der Elementarvorgänge zusammengereimt werden? Wie armselig steht der theoretische Physiker vor der Natur und vor - seinen Studenten!» ${ }^{5}$

Offenbar bezog sich Einsteins Bemerkung auf ein Experiment des Wiener Privatdozenten Erwin Schrödinger über die «Kohärenz von Licht in weitgeöffneten Bündeln», ${ }^{6}$ dessen Ergebnis im August 1919 in den Annalen der Physik erschienen war. Schrödinger hatte sich schon früher eingehend mit der Analyse der Schwankungserscheinungen beim radioaktiven Zerfall (den sogenannten Schweidlerschen Schwankungen) befaßt und hatte gehofft, seine Erfahrungen zum Nachweis der Einsteinschen «Nadelstrahlung» nutzbar machen zu können. Doch es stellte sich heraus, daß dieses Experiment als experimentum crucis für oder wider die Lichtquanten ungeeignet war.

Da man sich auch in München für die Lichtquanten zu interessieren begann, wurde Schrödinger im Novenber 1919 zu einem Vortrag im Münchner Mittwochskolloquium eingeladen. Arnold Sommerfeld hatte dort gerade sein berühmtes Werk über «Atombau und Spektrallinien» abgeschlossen, das eine Zusammenfassung seiner Vorlesungen während der Kriegsjahre darstellte. Als «die schwierigste und zugleich interessanteste Frage» der Atomphysik wurde darin das Verhältnis von Wellen- und Quantentheorie in einem abschließenden Kapitel behandelt. Noch vor zwei Jahren habe es aussichtslos geschienen, eine «Brücke von der alten Welt der Wellen zu der neuen Welt der Quanten» schlagen zu wollen. «Jetzt ist die Brücke hergestellt»...7

Ursache für diesen Stimmungsumschwung waren die erfolgreichen Überlegungen seines polnischen Mitarbeiters Adalbert Rubinowicz gewesen, dem kürzlich eine Herleitung der spektroskopischen Auswahl- und Polarisationsregeln durch Anwendung des Drehimpulssatzes auf das Gesamtsystem von Atom und Strahlungsfeld bei einem Übergangsprozeß gelungen war. Über die Einsteinsche Lichtquantentheorie hatte sich Sommerfeld in der ersten Auflage seines Buches dagegen nur mit großer Zurückhaltung geäußert. Daß in dieser Theorie «die Vorstellung der Kugelwelle keinen Platz» hatte, wurde von ihm als ein besonders schwerwiegender Mangel empfunden ${ }^{8}$.

Schrödingers Vortrag über «Einsteins Theorie der Nadelstrahlung» dürfte deshalb einen Anlaß zu anregenden Diskussionen mit den Mitgliedern des Sommerfeldschen Instituts gegeben haben. Unter diesen befand sich 
auch der junge Pauli, mit dem Schrödinger von nun an einen regen Gedankenaustausch unterhielt.

Obwohl Pauli damals noch Student im 3. Semester war, hatte sich die Kunde seiner außerordentlichen Begabung schon allgemein herumgesprochen. Sein Interesse, das anfangs fast ausschließlich den relativistischen Problemen galt, wandte sich um diese Zeit der Quantentheorie zu.

Besonders bei der Umgestaltung der angefügten Zusätze in der ein Jahr später erfolgenden zweiten Auflage seines Buches ließ Sommerfeld sich von Pauli helfen. Deshalb können wir davon ausgehen, daß die dort wiedergegebenen Äußerungen über die Lichtquanten auch Paulis Standpunkt mitenthalten. Insbesondere heißt es dort: «Die klassische Wellentheorie ist nicht ganz falsch und die Quantentheorie der Strahlung noch nicht ganz richtig; worauf es in Zukunft ankommt, ist, zwischen beiden die Brücke zu schlagen. Die energetische Auffassung der Größe $h v$ ist am furchtlosesten von Einstein verfochten ... Wie ist (der Umsatz von Strahlungsenergie des Betrages $h v$ in Hubarbeit bei der Loslösung eines Elektrons aus dem Atomverbande) zu verstehen, wenn die Wellenenergie nicht in Form von Lichtquanten konzentriert und auf einmal verfügbar ist? Andererseits ist die kontinuierliche Ausbreitung der Wellenenergie bei den Vorgängen der Interferenz und Beugung so sicher begründet, daß von hier aus die Lichtquantenvorstellung einfach als undiskutierbar erscheint. Die moderne Physik steht hier einstweilen zwischen unvereinbaren Gegensätzen und muß ein freimütiges cnon liquets bekennen.» ${ }^{9}$

Aber auch Einstein dachte weiterhin über «Experimente an der Grenze Wellen-Korpuskeln» nach ${ }^{10}$. In Gesprächen und Briefen hatte er bereits um 1920 die Idee der «Führungsfelder» in Umlauf gesetzt, die für die spätere Entwicklung eine so fundamentale Rolle spielen sollte ${ }^{11}$. In einem allgemeinverständlichen Aufsatz, den Walter Schottky im Juni 1921 veröffentlichte, behandelte er unter Berufung auf «eine persönliche Mitteilung von Herrn Prof. Einstein» die Widersprüche, die sich aus der Lichtquantenhypothese ergaben. Sofern an dieser Hypothese überhaupt etwas Wahres sei, schreibt Schottky, sollten diese Lichtquanten eine ziemlich große Ausdehnung besitzen; ein solcher Lichtknoten müsse gewissermaßen «geistige Fühler» nach allen Seiten ausstrecken und die Materie in seiner Umgebung abtasten, damit die Interferenzerscheinungen verständlich werden ${ }^{12}$. 


\section{Ein erster wellentheoretischer Lösungsversuch:}

\section{Die Theorie von Bohr, Kramers und Slater}

Der hier schon vorgeprägte Begriff des Führungsfeldes kam Anfang 1924 als virtuelles Strahlungsfeld in der Theorie von Bohr, Kramers und Slater neu zum Vorschein. Niels Bohr war damals noch ein überzeugter Anhänger der Wellentheorie des Lichtes. Ehrenfest berichtete Einstein Anfang 1922, daß Bohr viel eher bereit sei, den Energie- und Impulssatz für atomare Elementarprozesse aufzugeben, «als die Schuld auf den Äther zu schieben». ${ }^{13}$ Selbst den Compton-Effekt wollte Bohr nicht zugunsten der korpuskular theoretischen Deutung verstanden wissen ${ }^{14}$.

Um so freudiger begrüßte Bohr deshalb eine Anregung seines jungen amerikanischen Gastes John C. Slater, der sich vom Dezember 1923 bis zum Juni 1924 am Kopenhagener Institut aufhielt ${ }^{15}$. Danach sollte das Atom als ein System «virtueller» Oszillatoren betrachtet werden, deren Frequenzen den möglichen Quantenübergängen entsprachen und die über ein «virtuelles» Strahlungsfeld ständig mit den virtuellen Oszillatoren der benachbarten Atome in Verbindung standen.

«Das Aufregendste» an dieser Theorie war jedoch, wie es Schrödinger in einem Brief an Bohr ausdrückte, «die grundsätzliche Verletzung des Energie-Impulssatzes» und damit des kausalen Zusammenhanges bei jedem Strahlungsprozeß. ${ }^{16}$ Schrödinger gesteht auch zu, daß ihn «diese Wendung außerordentlich sympathisch» berühre. "Als Schüler des greisen Franz Exner» habe er sich schon lange «mit dem Gedanken befreundet, daß die Grundlage unserer Statistik wahrscheinlich nicht mikroskopische «Gesetzmäßigkeit), sondern vielleicht ‘absoluter Zufall〉 bildet, und daß vielleicht sogar Energie- und Impulssätze nur statistische Geltung haben.» Was Schrödinger an der neuen Strahlungstheorie jedoch am meisten zusagte, war eine «weitgehende Rückkehr zur klassischen Theorie, was die Strahlung anbetrifft».

Noch deutlicher als hier hatte Schrödinger seinen Standpunkt zur Kausalitätsfrage kurz zuvor in einem Schreiben an Hans Reichenbach dargelegt: «Warum erwarten wir unter völlig gleichen Umständen stets auch den völlig gleichen Erfolg? ... Warum drängt sich uns bei verändertem Erfolg ganz unabweislich die Überzeugung auf, es müßten sich auch die Umstände, wenn auch noch so wenig, geändert haben? ... Ich nenne das das Rätsel des Induktionsschlusses. Ich glaube nicht, daß es für uns im eigentlichen Sinn lösbar ist.» ${ }^{17}$ 
Interessant im Hinblick auf die spätere Diskussion sind auch die Vermutungen, die Schrödinger bei dieser Gelegenheit über die Form der künftigen Gesetze des atomistischen Geschehens anstellte. Er nimmt an, daß scharf bestimmten Umständen ein ganzes Kontinuum möglicher Erfolge entsprechen könnte.

Schrödinger war also damals bereit, das Kausalitätsprinzip im atomaren Einzelvorgang zu opfern. Von den unanschaulichen «Quantensprüngen» wollte er aber nichts wissen.

Besonders die Gruppe der jüngeren und progressiveren Physiker wie Pauli, Heisenberg, Jordan und Dirac war aber inzwischen davon überzeugt, daß der Zugang zu einer neuen Quantentheorie nur durch einen weitgehenden Verzicht auf die anschauliche Vorstellungswelt der klassischen Physik zu erzielen war. Doch auch in dieser Gruppe waren die Beweggründe sehr unterschiedlich. Werner Heisenberg - damals unter dem direkten Einfluß von Bohr stehend - glaubte noch an eine korrespondenzmäßige Entsprechung aller Quanteneffekte in der klassischen Theorie. Für ihn war deshalb ein Ausgang des Bothe-Geiger-Experiments in beiderlei Richtung annehmbar $^{18}$.

Pauli dagegen lehnte die neue Theorie von Anfang an ab, und selbst Niels Bohr vermochte ihn nur vorübergehend umzustimmen. Er sagte, sein «wissenschaftliches Gewissen lehne sich ... gegen diese Auffassung stark auf.» Pauli gab allerdings zu, daß es sich dabei immer nur um Gefühlsargumente» handelte, «weil man logisch da nichts beweisen kann, und daß auch die vorliegenden Erfahrungsergebnisse nicht ausreichen, um für oder gegen Bohrs Auffassung zu entscheiden». ${ }^{19}$

Auf der Naturforscherversammlung in Innsbruck führte Pauli im Herbst 1924 zum erstenmal ein längeres Gespräch mit Einstein. Als er ihn um seine Meinung zur Strahlungstheorie befragte, machte Einstein zahlreiche Einwände ${ }^{20}$. Einstein war «überzeugt, daß die weitere Entwicklung dahin führen werde, einem Lichtquant als Träger von Energie und Impuls eine höhere Realität zuzuschreiben als dem Wellenfeld. Von dem Moment an, wo man es aufgegeben habe, daß die Frequenz des Lichtes übereinstimmen muß mit der Frequenz des emittierenden Elektrons, habe für ihn gefühlsmäßig dem undulatorischen Charakter des Lichtes etwas Schattenhaftes angehaftet.» Aber «zu einem Verzicht auf die strenge Kausalität mochte [Einstein] sich nicht treiben lassen, bevor man sich nicht noch ganz anders dagegen gewehrt hat als bisher». ${ }^{21}$

Unabhängig von den Meinungsverschiedenheiten dieser Autoritäten 
begann Pauli immer energischer die Kopenhagener «Irrlehre» zu bekämpfen. Die Frage nach der statistischen Korrelation der Quantenprozesse beunruhigte ihn jetzt weniger als die zu einseitig auf eine raum-zeitliche Beschreibung der Bewegungsvorgänge gerichtete Tendenz der Kopenhagener Physik ${ }^{22}$. Pauli glaubte schon damals, daß der Begriff der Elektronenbahnen im Atom ebenso unzulässig sei wie bei den Lichtquanten, bei denen diese Vorstellung bekanntlich mit den beobachteten Interferenzerscheinungen nicht in Einklang zu bringen war ${ }^{23}$.

Bis zur endgültigen Widerlegung der Strahlungstheorie durch das Experiment von Bothe und Geiger hatten viele Physiker ihre Hoffnung auf eine Lösung der Quantenprobleme aus dieser Richtung gesetzt. Doch die Vertreter des extremen Quantenstandpunktes, die in der Diskontinuität der mikrophysikalischen Prozesse ihr eigentliches Wesen erblickten, blieben skeptisch. Schon im Dezember 1924 konnte Einstein seinem holländischen Kollegen Lorentz von dem Ausgang des Experiments von Bothe und Geiger berichten, «welches für die strikten Lichtquanten und gegen die Ansichten spricht, welche Bohr-Kramers-Slater neulich aufgeführt haben. Sie zeigten, daß beim Compton-Effekt abgebeugte Strahlung und nach der anderen Seite ausgeschleudertes Elektron statistisch voneinander abhängige Ereignisse seien. Das Energie-Impuls-Prinzip scheint aber doch streng und nicht nur statistisch zu gelten.» ${ }^{24}$ Ehrenfest wertete dieses bereits von ihm erwartete Ergebnis als einen «Triumph von Einstein über Bohr». ${ }^{25}$ Pauli sah darin einen deutlichen Hinweis auf die physikalische Zweckmäßigkeit der Lichtquantenvorstellung, der er keine geringere Realität zusprechen mochte als dem Elektronenbegriff ${ }^{26}$.

Auch Niels Bohr hatte sich bereits vor der öffentlichen Bekanntgabe des Resultates - «nach vieler Diskussion und namentlich unter dem katalytischen Einfluß von Pauli», wie Kronig berichtete ${ }^{27}$ - von der Strahlungstheorie zu distanzieren begonnen. Nun «ist wohl nichts anderes zu tun», stellte er Ende März 1925 resignierend fest, «als unseren Revolutionsversuch möglichst schmerzlos in Vergessenheit zu bringen». ${ }^{28}$

\section{Die Heisenbergsche «Höllenmaschine»}

Vor diesem Hintergrund entwickelte Heisenberg im Sommer 1925 seine neue Quantenmechanik, die sogleich Paulis begeisterten Zuspruch fand. «Überhaupt glaube ich», schrieb Pauli, nachdem Heisenberg ihm, von Helgoland 
kommend, seine «Quantenmechanik» erläutert hatte, «daß ich jetzt hinsichtlich meiner wissenschaftlichen Ansichten Heisenberg sehr nahe gekommen bin und daß wir ziemlich in allem übereinstimmende Meinungen haben.» ${ }^{29}$

Diese Quantenmechanik verkörperte das von den Quantenphysikern geforderte Ideal, denn sie verzichtete weitgehend auf die Hilfsmittel und Modellvorstellungen der älteren Atomtheorie. Von Ort, Geschwindigkeit oder anderen mechanischen Bestimmungsstücken eines Teilchens war jetzt nirgends mehr die Rede ${ }^{30}$. Deshalb wurde Heisenbergs Arbeit, trotz der Bewunderung und des großen Aufsehens, das sie sofort erregte, von den meisten Physikern der älteren Generation mit größtem Mißtrauen aufgenommen ${ }^{31}$. Als Einstein sie nach der Sommerpause in Berlin kennenlernte, kommentierte er mit leichter Ironie: «Heisenberg hat ein großes Quantenei gelegt. In Göttingen glauben sie daran, (ich nicht)». ${ }^{32}$ Später, als sich bereits die Kunde von Schrödingers großem Erfolg herumgesprochen hatte, meinte Einstein sogar, daß «die Born-Heisenberg-Sache wohl doch nicht richtig sein» wird. ${ }^{33}$

Wie den meisten anderen bereitete der abstrakte mathematische Formalismus der Heisenbergschen Mechanik auch Einstein große Schwierigkeiten, und er sprach gerne von einem «Hexeneinmaleins» oder von einer «Höllenmaschine», wenn er auf sie zu sprechen kam. Ähnlich urteilte er über Diracs Arbeiten, die er «im Einzelnen überhaupt nicht verstehen konnte» ${ }^{34}$ und mit einem «Balancieren auf schwindelndem Pfad zwischen Genie und Wahnsinn» verglich. «Nichts, was man herzhaft mit Händen greifen kann.» ${ }^{35}$

Doch schon die ersten praktischen Anwendungen der Quantenmechanik zeigten ihre Überlegenheit gegenüber der älteren Theorie. Sie führten nicht nur zu bekannten und neuen Lösungen, sondern sie machten auch viele der bisherigen künstlichen Zusatzannahmen überflüssig.

Die Quantenmechaniker konnten also voller Zuversicht die Richtigkeit ihres Verfahrens proklamieren, während bei den Kritikern vorübergehend große Hilflosigkeit herrschte. Die Schwierigkeiten, mit denen beispielsweise die damals aufkommende Spinhypothese zu kämpfen hatte, weil sie in einer anschaulichen Vorstellung begründet war, werden nur in diesem Zusammenhang verständlich. 


\section{Schrödingers Alternative}

Wie sehr die verschiedenen Physikergruppen in ihren eigenen Gedankenkreisen gefangen waren, zeigt auch die unterschiedliche Aufnahme der Idee der Materiewellen, die Louis de Broglie bereits seit 1923 propagiert hatte. Während Einstein und Schrödinger darauf ansprachen ${ }^{36}$, wurde Max Born trotz der Arbeiten, die Walter Elsasser in seiner unmittelbaren Umgebung ausführte - erst durch Paul Ehrenfest «ein Licht aufgesteckt. - Darauf habe ich die Arbeit von Louis de Broglie gelesen», schrieb er Einstein im Juli 1925, «und bin allmählich auch hinter Deine Schliche gekommen. Jetzt glaube ich, daß die «Wellentheorie der Materie〉 eine sehr gewichtige Sache werden kann.» ${ }^{37}$

Für die begriffliche Entwicklung der Quantentheorie war es deshalb von außerordentlicher Bedeutung, daß Schrödinger schon im Abstand von wenigen Monaten eine Wellenmechanik vorlegen konnte, die zunächst eine Rückkehr in die anschauliche Vorstellungswelt der älteren Physik versprach. Da die Schrödingersche Theorie die Leistungsfähigkeit der Quantenmechanik sogar noch zu übertreffen schien, entspann sich vorübergehend ein Konkurrenzkampf zwischen den Anhängern beider Theorien, der erst abflaute, nachdem die vollständige Gleichberechtigung der beiden Theorien offenkundig war.

Schrödinger war anfangs sehr zuversichtlich, «daß es jetzt gelingen wird, eine harmonische, von allen Härten freie Quantentheorie aufzubauen, und zwar nicht in dem Sinne, daß alles immer unstetiger und ganzzahliger wird, sondern gerade im umgekehrten Sinn: die schönen klassischen Methoden liefern selbsttätig alle Ganzzahligkeit». ${ }^{38}$ Zuerst hatte Schrödinger geglaubt, die $\Psi$-Funktion als einen mechanischen Schwingungsvorgang und die von einem Atom emittierten Frequenzen als Differenztöne der entsprechenden Eigenschwingungen deuten zu können. Gerade diesen anschaulichen Zug seiner Theorie betrachtete er als einen der wesentlichsten Vorzüge gegenüber der Methode von Heisenberg, Born und Jordan ${ }^{39}$.

Auch Wilhelm Wien, der mit zu den Befürwortern der Schrödingerschen Auffassung gehörte, glaubte, daß «das Problem der Atomschwingungen gelöst sei, und zwar erfreulicherweise im engsten Anschluß an die klassische Theorie». Ebenso waren Planck und Einstein ganz begeistert und «der gleichen Meinung»s. ${ }^{40}$

Pauli, der damals gerade seinen Widerstand gegen den Elektronenspin aufgegeben hatte und über die Osterferien von Hamburg nach Kopenhagen 
gekommen war, beteiligte sich sofort an der Diskussion der Schrödingerschen Theorie, die er «zu dem Bedeutendsten zählte, was in letzter Zeit geschrieben wurde». ${ }^{41}$ Obwohl Pauli anfangs noch die Deutung der Differenzschwingungen als Lichtwellen akzeptierte, glaubte er grundsätzlich nicht an eine Rückkehr zu einer raum-zeitlichen Beschreibung der Elektronenbewegung durch Schrödingers Ansatz.

Um diese Zeit begann sich ein engeres persönliches Verhältnis zwischen Pauli und Schrödinger anzubahnen, wie jetzt die vertraulichere Anrede «Du» in den Briefen erkennen läßt.

Eine erste öffentliche Aussprache zwischen den sogenannten «Matriziern» und «Wellenmechanikern» fand Ende Juni 1926 während der physikalischen Vortragswoche in Zürich statt, die von Peter Debye und Paul Scherrer veranstaltet wurde. Außer Sommerfeld waren auch Pauli und Otto Stern aus Hamburg zugegen. Vergeblich versuchten sie, Schrödinger von der Unhaltbarkeit seiner anschaulichen Deutung abzubringen. Obwohl Schrödinger inzwischen die naiv-anschauliche Auffassung seiner « $\Psi$-Schwingungen» fallen gelassen und durch eine elektrodynamische Hypothese ersetzt hatte ${ }^{42}$, so war er doch keinesfalls bereit, ihren Wirklichkeitscharakter aufzugeben. - Noch während die Zürcher Veranstaltung tagte, reichte Max Born seine bekannte Stoßarbeit ein, die unter Rückgriff auf Einsteins Gespensterfeld die statistische Interpretation der Wellenfunktion begründete. Damit war auf Kosten des strengen Kausalprinzips die Grundlage für eine logisch unanfechtbare Beschreibung geschaffen, mit der nun Schrödingers widersprüchliche Deutungsversuche nicht konkurrieren konnten.

Die Nachricht von Schrödingers Erfolg hatte sich rasch verbreitet ${ }^{43}$. Bald folgten die ersten Einladungen. Die erste größere Vortragsreise, die er im Juli 1926 unternahm, galt natürlich Berlin, wo damals auch die größte Zahl eminenter Physiker jener Zeit versammelt war ${ }^{44}$. Hier fand Schrödinger in Einstein, Planck und von Laue einen ähnlich eingestellten Personenkreis vor, und so hatte er wenig Kritik zu befürchten ${ }^{45}$. Max von Laue war so sehr von Schrödingers Ausführungen angetan, daß er «seit August fast ausschließlich Quantentheorie trieb». Schrödingers Methode sagte ihm viel mehr zu als die «geradezu entsetzliche Paulische Balmer-Arbeit.» ${ }^{46}$ Doch bei näherem Hinsehen kamen bei Einstein erste Bedenken auf, die er wenig später Ehrenfest übermittelte. Besonders «schwer verdaulich» für das anschauliche Verständnis empfand er «die Wellen im n-dimensionalen Koordinatenraum». «Hic Wellen, Hic Quanten! beider Realität steht felsenfest. Aber der Teufel macht einen Vers darauf (der sich wirklich reimt).» ${ }^{47}$ 
Eine ganz andere Stimmung erwartete Schrödinger in München, wo er auf der Rückreise von Berlin haltmachte. Heisenberg, der inzwischen wieder bei Bohr in Kopenhagen arbeitete, hielt sich während der Sommerferien in München auf und war zu Schrödingers Vortrag ins Sommerfeldsche Mittwochskolloquim gekommen. Er hatte natürlich zahlreiche Einwände: «So nett Schrödinger persönlich ist, so merkwürdig find' ich seine Physik», urteilte er über den Vortrag. «Schrödinger wirft ja alles «Quantentheoretische»: nämlich lichtelektrischen Effekt, Francksche Stöße, Stern-Gerlacheffekt usw. über Bord; dann ist es nicht schwer, eine Theorie zu machen. Aber sie stimmt eben nicht mit der Erfahrung.» ${ }^{48}$ Besonders Schrödingers Versuch, die von einem Atom abgestrahlten Frequenzen durch ein Überlagerungsphänomen von je zwei zugleich angeregten Eigenschwingungen zu erklären, erregte Heisenbergs und Sommerfelds Widerspruch ${ }^{49}$.

«Daß der lichtelektrische Effekt die größte begriffliche Schwierigkeit für die Durchführung einer klassizistischen Theorie darbietet», wurde nun auch von Schrödinger zugestanden. Aber «Bohrs Standpunkt, eine räumlichzeitliche Beschreibung sei unmöglich, lehen ich a limine ab. ... Der Zweck der Atomforschung ist, unsere diesbezüglichen Erfahrungen unserem übrigen Denken einzufügen; dieses ganze übrige Denken bewegt sich, soweit es die Außenwelt betrifft, in Raum und Zeit. Gelingt also die Einfügung in Raum und Zeit nicht, so ist der ganze Zweck verfehlt.» ${ }^{50}$

\section{Auf der Suche nach einem festen Fundament für die «Diskontinuumswelt»}

In seinem Referat über Quantenmechanik während der Düsseldorfer Naturforscherversammlung im Herbst 1926 hatte Heisenberg Schrödingers Theorie öffentlich kritisiert. Er würdigte zwar ihre mathematische Brauchbarkeit, lehnte aber die von Schrödinger behauptete Möglichkeit einer kontinuierlichen Beschreibung der Quantenphänomene grundsätzlich ab. Noch schärfer war dieser Aspekt in Jordans Schriften und Briefen betont worden ${ }^{51}$.

Auch Schrödingers Diskussion mit Bohr und Heisenberg im Oktober 1926 in Kopenhagen führten zu keiner Annäherung der Standpunkte. Am stärksten gingen die Meinungen über die statistische Bedeutung der $\Psi$ Wellen auseinander, die in gewissem Sinne eine Wiederbelebung der BohrKramers-Slater-Theorie beinhaltete. Ausgangspunkt der Diskussion war ein quantenmechanisches System im Grundzustand, das durch eine Störung 
in eine Anzahl verschiedener Eigenschwingungen überführt wird. Während aus der Sicht der «Quantenmechaniker» die entsprechenden Partialamplituden nur ein Maß für die Wahrscheinlichkeiten sein sollten, das System am Ende in einem dieser Zustände zu finden, glaubte Schrödinger ursprünglich, daß alle diese Eigenschwingungen gleichzeitig bei einem Einzelsystem angeregt sein könnten ${ }^{52}$.

Auch Pauli glaubte, daß nur eine statistische Verknüpfung der Wellenfunktion mit den physikalischen Meßergebnissen mit den Tatsachen zu vereinbaren sei. In einem Nachtrag zu seinem Quantenartikel im MüllerPouilletschen Lehrbuch begründete er seinen Standpunkt: Gegen Schrödingers anschauliche Deutung der $\Psi$-Funktion im Sinne einer Kontinuumsphysik führt er das Zerfließen der Wellenpakete an, die der allenthalben bestätigten Lokalisierung der Masseteilchen widerspreche. Auch der Gültigkeit der Erhaltungssätze von Ladung, Energie und Impuls beim Einzelprozeß und das Auftreten der Eigenfunktionen in mehrdimensionalen Räumen spreche gegen Schrödingers Standpunkt ${ }^{53}$.

Schließlich, nach vielen Diskussionen, gab Schrödinger im November zu, daß die Schwierigkeiten, die seine «Dichtehypothese» mit sich brachte, durch die Bornsche Deutung «wie mit einem Schlag behoben scheinen.... In der Tat will ja auch Bohr die ganzen Wellen, Maxwellsche und de Brogliesche, als 〈Gespensterfeld〉 beibehalten», erklärte er weiter. ... Es wird vielleicht auf derlei hinauskommen, aber der Gedanke ist noch lange nicht so weit zu Ende gedacht, wie man in Göttingen glauben machen will. Hinten und vorne ist alles unklar, die Leute können auf keine Frage antworten, die im Jargon des gesunden Menschenverstandes gestellt wird.» ${ }^{54}$

Doch die allgemein sichtbaren Erfolge der mit vertrauten mathematischen Hilfsmitteln operierenden Schrödingerschen Theorie und die bestechende Anschaulichkeit, die ihr Schrödinger beilegte, erregte das Mißtrauen der Göttiger Phyiker. Sie befürchteten, wie Jordan begründete, daß er seine Ergebnisse «nicht zur quantitativen Verschärfung, sondern zur Überwindung der Bohrschen Theorie» benutzen könnte ${ }^{55}$. Als Schrödinger sich daraufhin bei Born über diese Ausfälle seines jungen Mitarbeiters beschwerte, erklärte Born: «Bei meinen jüngeren Mitarbeitern (offenbar meinte Born insbesondere Jordan, Wigner, von Neumann, Oppenheimer und London) herrscht Kampfstimmung, weil der überwältigende Sieg der Wellenmechanik uns sachlich ungerechtfertigt erscheint und darauf zurückgeführt wird, daß sie dem Bedürfnis der meisten nach Rückkehr zu klassischen Formen der Physik entgegenkommt.» ${ }^{56}$ 
Mit um so größerem Eifer nutzten deshalb die «Göttinger» jetzt jede Gelegenheit, die Schwächen der Schrödingerschen Auffassungen öffentlich herauszustellen. «Da Schrödinger uns angegriffen hat», sagte Heisenberg, habe er nichts dagegen, daß Jordan sich in eine Polemik mit ihm einlasse ${ }^{57}$. In seinem «Kampf für die Matrizen und gegen die Wellen» geriet Heisenberg sogar selbst zeitweilig in einen Konflikt mit Bohr ${ }^{58}$.

Pauli nahm damals eine mehr vermittelnde Stellung zwischen den «Matriziern» und den «Herren von der Kontinuumstheorie» ${ }^{59}$ ein. Dennoch konnte er es nicht unterlassen, Schrödinger seinen «Zürcher Lokalaberglauben» vorzuhalten ${ }^{60}$.

Diese von Pauli einmal als regressiv und progressiv bezeichneten Bestrebungen in der damaligen Quantenphysik haben ohne Zweifel die begriffliche Klärung des quanten-mechanischen Formalismus beschleunigt.

Schrödingers Auffassungen konnte man am wirksamsten entgegentreten, wenn es gelang, die Begriffe von Ort und Geschwindigkeit in einer quantenmechanischen «Diskontinuumswelt» neu zu fassen. Ein entscheidender Fortschritt war zunächst die Verallgemeinerung der Bornschen Wahrscheinlichkeitsdeutung durch Pauli. «Pauli schrieb mir», berichtete Heisenberg im November 1926, «daß er über die Bornschen Stoßprobleme Neues gefunden habe: man kann beweisen, daß nach der Matrizenrechnung immer dasselbe herauskommt wie bei Born. Außerdem ist Born und Schrödinger nur Spezialfall allgemeinerer Gesetze. Man kann z.B. auch Wellen im p-Raum konstruieren und damit die Eigenwerte ausrechnen, oder solche im I-Raum, w-Raum, ganz wie es zweckmäßig ist.» ${ }^{61}$ Formal ausgestaltet wurde dieser Sachverhalt im Dezember 1926 durch die DiracJordansche Transformationstheorie. Damit war auch die der Schrödingerschen Theorie zugrunde liegende Bevorzugung des gewöhnlichen Raumes aufgehoben.

Diese Bemühungen gipfelten bekanntlich in Heisenbergs Unsicherheitsrelationen, die er Pauli schon Ende Februar 1927 in einem 14 Seiten langen Schreiben mitteilen konnte. Erleichtert stellte Heisenberg wenig später fest, «seit einem Jahr langen Schwimmen in kontinuierlichem trüben Wasser» fühle er nun «wieder festen diskontinuierlichen Grund unter den Füßen. Der Übergang von Mikro- zu Makromechanik kann natürlich jetzt auch sozusagen «stehend freihändig» ausgeführt werden ...» ${ }^{62}$

Heisenberg reichte diese Ergebnisse kurz darauf - noch während Bohrs Abwesenheit von Kopenhagen - zur Veröffentlichung ein. Bohr mißbilligte nach seiner Rückkehr, daß Heisenberg in seiner Darstellung Schrödingers 
Verdienste bei dieser Begriffsklärung nicht erwähnte und der Wellenmechanik sogar den Vorwurf machte, sie habe durch ihre populäre Anschaulichkeit vom geraden Weg abgeführt. Durch Betonung der komplementären Gegensätze - den Welle-Teilchen-Dualismus betrachtete Bohr dabei nur als einen Spezialfall seines allgemeineren Prinzips - gelang es Bohr schließlich, eine Verschmelzung der beiden Standpunkte herzustellen, die unter dem Namen der Kopenhagener Deutung in die Geschichte eingegangen ist ${ }^{63}$.

So war es schließlich innerhalb der kurzen Zeitspanne von nur einem Jahr gelungen, die gewünschte «Synthese der Quanten- und Wellenauffassung» 64 durch die Ansätze von Schrödinger, Born, Pauli, Heisenberg, Bohr, Jordan und Dirac zu erreichen und unter einem gemeinsamen Dach zu vereinigen. Natürlich blieben einige Spannungen weiterhin bestehen. Besonders Schrödinger und Born grollten den Kopenhagenern, weil in der Kopenhagener Formulierung ihr Anteil an dieser Entwicklung nicht deutlich genug zum Ausdruck kam. «Was die «dänische Theorie» anlangt», äußerte Born in späteren Jahren, «so hätte ich allen Grund, ihren Verkündern feindlich zu sein. Denn sie haben ein gut Stück dessen, was ich zur Quantentheorie beigetragen habe, (nostrifiziert), wie wir früher in Göttingen zu sagen pflegten. Dies hat mich oftmals in Wut versetzt, aber nicht verhindert, anzuerkennen, was Bohr Positives geleistet hat.» ${ }^{65}$

Die Vorträge und Diskussionen während der Volta-Feier in Como und auf dem Solvay-Kongreß in Brüssel im Herbst 1927 dienten im wesentlichen nur noch der weiteren Ausarbeitung dieser Ideen. Eine besondere Verfeinerung erhielt diese Deutung allerdings noch durch Heisenbergs Definition des «Schnittes», der «den feinen Zusammenhang der Erscheinungen zerstört; an der Stelle, wo wir den Schnitt zwischen dem zu beobachtenden System einerseits, den Apparaten andererseits machen, müssen wir mit Schwierigkeiten für unsere Anschauung rechnen», sagte Heisenberg. Hierauf aufbauend, entwickelte er dann die Vorstellung der Zustandsreduktion durch einen Beobachtungsakt, abermals auf Schrödingers ursprünglich mehr anschaulichen Begriff der Wellenpakete zurückgreifend: «Die Wellen haben nicht die unmittelbare Realität, die wir früher den Wellen der Maxwellschen Theorie zugeschrieben haben. Man muß sie als Wahrscheinlichkeitswellen deuten und daher plötzliche Änderungen bei jeder neuen Beobachtung erwarten.» ${ }^{66}$

Die ursprünglich von Max Born noch für möglich gehaltene Einführung von Mikrokoordinaten, «um die Determiniertheit des Einzelprozesses wenigstens im Prinzip zu retten», ${ }^{67}$ schien jetzt durch Johann von Neumanns 
Beweis ausgeschlossen zu sein. Obwohl dieser Sachverhalt erst 1932 durch von Neumanns bekanntes Buch allgemein zugänglich wurde, war man in Göttingen schon 1928 damit vertraut ${ }^{68}$.

Für die unmittelbar an der Entwicklung der Quantenmechanik beteiligten Physiker wurde damit die nicht relativistische Quantenmechanik formal wie auch inhaltlich als eine abgeschlossene Theorie angesehen.

Selbst Schrödinger, der anfangs so zäh für eine Rückkehr zu einer Kontinuumstheorie gefochten hatte, gab schließlich «mehr und mehr zu, daß sein reiner Elektromagnetismus mit $\varrho=\psi \bar{\psi}$ undurchführbar» sei $^{69}$.

\section{Die Berliner Opposition}

Zum Wintersemester 1927/28 wurde Schrödinger zum Nachfolger von Max Planck nach Berlin berufen. Hier kam er in einen Kreis kongenialer Physiker wie Einstein, von Laue und Max Planck, die der neueren Entwicklung der Quantenmechanik weiterhin mit einer gewissen Skepsis gegenüberstanden. Als sogar Schrödinger in seiner Antrittsrede vor der Preußischen Akademie den absoluten Determinismus in Frage zu stellen wagte, konnte Max Planck in seiner Erwiderung «der Verlockung nicht widerstehen, hier einmal eine Lanze für die streng kausale Physik einzulegen». ${ }^{70}$

Auch Einstein konnte sich trotz seiner Bewunderung für die «wirklich imponierenden neuen Resultate auf dem Gebiete der Quanten ... nicht mit der Vogelstrauß-Anschauung des halb-kausalen und halb-geometrischen befreunden», wie er Anfang 1927 erklärte ${ }^{71}$. Noch weniger behagte ihm die statistische Deutung, was er durch seinen bekannten Ausspruch vom würfelnden Gott zum Ausdruck gab. Während des Solvay-Kongresses hielt ihm Niels Bohr entgegen, schon die alten jüdischen Propheten hätten die Schwierigkeiten gekannt, Gottes Natur durch menschliche Begriffe zu erfassen $^{72}$.

Auch nach Aufgabe seiner ersten anschaulichen Deutungen hat Schrödinger keineswegs die Kopenhagener Interpretation akzeptiert. Für ihn war damit - wie er in seinen Aufsätzen immer wieder betonte - das eigentliche Problem dieser «Zentaurennatur» ${ }^{73}$ der Materie noch nicht gelöst. «Die Begriffe müssen aufgegeben werden», forderte er, «nicht ihre scharfe Definiertheit.» ${ }^{74}$ «Eine experimentelle Entscheidung des Dilemmas», ob das Verhalten des Atoms in jedem Einzelfalle streng kausal determiniert sei, ... dürfte kaum möglich sein, weil eben, rein denktechnisch, sowohl das 
Gesetz auf Zufall als auch der Zufall auf Gesetz zurückgeführt werden kann, wenn man Lust dazu hat.» ${ }^{75}$

Neben diesen erkenntnistheoretischen Bedenken waren öfters auch die Schwierigkeiten im Umgang mit dem mathematischen Bedenken waren öfters auch die Schwierigkeiten im Umgang mit dem mathematischen Formalismus der Matrizen- und Gruppentheorie, dessen sich die neue Quantenmechanik bediente, daran schuld, daß gerade bei der älteren Physikergeneration diese Abneigung entstand. Hermann Weyls berühmtes Werk über «Gruppentheorie und Quantenmechanik» erregte Schrödingers Unwillen. «Liebe Mathematiker, wir wissen, wie bitter nötig wir vieles von dem hätten, was Ihr uns zu sagen habt», schrieb er an Hermann Weyl. «Gebt Euch, bitte, Mühe, uns das in leichter, faßlicher Form und mit nicht gar zu vielen neuen Begriffsbildungen zu sagen - möglichst in schäbigen, alten abgetragenen Begriffen, die Euch schon langweilig sind, ich weiß es. Neue Begriffsgebäude aufbauen macht Spaß, es ist Eure allerureigenste Sphäre, aber für uns liegt das Physikalische noch viel zu tief im Dunkel, als daß wir hoffen könnten, in dieser Finsternis mit solchen komplizierten, ungewohnten Instrumenten erfolgreich arbeiten zu können ... Aber dem vollen Verständnis können wir uns erst nähern, wenn einfache, von einem menschlichen Gehirn mit einem Blick umspannbare Ideen die Totalität dessen, was bis jetzt vorliegt, erfassen.» ${ }^{76}$

Einstein nannte die Heisenbergsche Matrizenmechanik damals ein «Hexeneinmaleins», Ehrenfest zweifelte angesichts der großen Fülle von gruppentheoretischen Arbeiten in der Quantenmechanik, ob er «jemals die Gruppomanen capieren werde», ${ }^{77}$ und auch Schrödinger klagte über die Schwierigkeiten, die ihm die Arbeiten von Heisenberg und Dirac bereiteten ${ }^{78}$. Ehrenfest geriet darüber sogar in einen Depressionszustand, wie er Schrödinger im September 1930 mitteilte ${ }^{79}$. Er faßte seine Zweifel in seinen berühmten «Erkundigungsfragen» zusammen und sandte einen Korrekturbogen an Schrödinger. Die Fragen waren an jemand gerichtet, «der diese Materie wirklich beherrscht» und «in einer auch uns älteren Physikern lesbaren Form darzustellen» vermag ${ }^{80}$.

Dieses war eine der wenigen kritischen Stellungnahmen zur Quantenmechanik durch einen prominenten Physiker, der nicht unmittelbar dem Schrödingerschen Gedankenkreis angehörte.

"Mir war Ihr Brief Balsam, Balsam, Balsam», erwiderte Schrödinger. «Immer, immer wieder muß ich hier, wenn ich mit den hochbegabten Jungen spreche (wie Neumann, Wigner, London usw.) das Gefühl haben: sie ver- 
stehen nicht, was mir an dem üppig aufschießenden Theorienwalde der letzten Jahre so ganz und gar unerträglich ist. Sie sind zu höflich (leider!), es ausdrücklich zu sagen, aber im Herzen glauben sie immer doch, die Anpassung an die ganz neuen Rechen- und Denkmethoden, die Loslösung von dem alten fällt mir zu schwer. Wenn ich auch einer von denen sei, die da einmal einen guten Fund gemacht und ihr Teil zu der neueren Entwicklung beigetragen haben, so sei ich doch offenbar schon zu alt und zu wenig mathematisch (vielleicht auch philosophisch) veranlagt, um alle Konsequenzen zu ziehen und mich restlos an der Herrlichkeit des neuen Weltbildes zu erfreuen.» ${ }^{81}$

Obwohl Schrödinger im weiteren Verlauf seines Briefes auch auf einige der von Ehrenfest aufgezählten Probleme einging, hat er keine wirkliche Beantwortung derselben gegeben. Diese Antwort war Pauli vorbehalten, der sie natürlich ganz im Geiste der Kopenhagener Deutung behandelte.

Diejenigen Physiker, welche die Kopenhagener Deutung schon von Anfang an als ein Provisorium angesehen hatten, ${ }^{82}$ wurden durch diesen Vorstoß zu einer öffentlichen Aussprache ermuntert.

Zu ihnen gehörte auch Max von Laue, der ebenso wie Einstein die strenge Kausalität vertrat. In einem Beitrag zur Feier des 70. Geburtstages des langjährigen Herausgebers der Zeitschrift «Die Naturwissenschaften», Arnold Berliner, ergriff er die Gelegenheit, «Bohrs oft betonten «Dualismus» von Korpuskular- und Wellenauffassung» anzugreifen, indem er feststellte, daß sie «wohl mehr den W unsch nach der notwendigen Synthese» ausdrücke als sie tatsächlich zu vollziehen ${ }^{83}$.

Als von Laue zwei Jahre später abermals versuchte, den endgültigen Charakter der durch die Ungenauigkeitsrelationen geschaffenen Situation anzutasten und die statistische Grundlage der Quantenmechanik in Analogie zu derjenigen der statistischen Mechanik setzen wollte, soll Otto Stern entrüstet ausgerufen haben: «Sie sind ja noch reaktionärer als Einstein.» ${ }^{84}$

Um so freudiger wurde natürlich von Laues Stellungnahme durch Schrödinger begrüßt, der, inzwischen Einsteins Beispiel folgend, im Sommer 1933 ins Exil gegangen war.

Der politische Umschwung im Deutschen Reich hatte natürlich auch seine Konsequenzen in der Wissenschaft. Ganz besonders betroffen davon war die theoretische Physik, die nicht nur viele ihrer führenden Köpfe verlor, sondern auch als Disziplin nicht mehr die volle Anerkennung genoß. Dieser Umstand wirkte sich auch direkt auf die Austragung einer sich anbahnenden 
erkenntnistheoretischen Diskussion über die Grundlagen der Quantenmechanik aus.

Arnold Berliner war als Schriftführer der «Naturwissenschaften» und als enger Freund von Einstein, Schrödinger, von Laue und Ehrenfest natürlich mit den inneren Konflikten über die erkenntnistheoretische Position in der Quantenmechanik vertraut. Da seine Zeitschrift jetzt unter den neuen politischen Verhältnissen ohnehin der Kritik von Gehrck und Stark ausgesetzt war, weil er «jahrzehntelang die Einsteinlehre propagiert und jede andere Meinung von der Diskussion ausgeschlossen» habe ${ }^{85}$, suchte er im Sommer 1935 den allgemeinen Verfall seiner Zeitschrift noch einmal durch eine öffentliche Polemik unter Beteiligung aller theoretischen Physiker von Rang aufzuhalten ${ }^{86}$. Als willkommene Gelegenheit bot sich ihm der im «Physical Review» veröffentlichte Artikel von Einstein, Podolsky und Rosen an. Schrödinger erklärte sich sofort bereit, an der Diskussion teilzunehmen. Da eine solche Polemik natürlich nur unter Beteiligung beider Parteien in Gang kommen konnte, richtete Berliner gelegentlich einer anderen Korrespondenz mit Paulis damaligem Assistenten Weisskopf an diesen die Frage, was Pauli zu dem Aufsatz im «Physical Review» meine. Daraufhin «kam von Pauli selber eine Karte», berichtete Berliner, «in der er sich mit der ihm eigenen Schärfe gegen den Aufsatz ausspricht, Laues Stellung zur ganzen Quantenmechanik mit höchster Skepsis beurteilt und von mir erwartet, daß ich auch Heisenberg an der Angelegenheit zu Rate ziehe.» Die Fauststelle zitierend: «Wie schien mir's schwarz, und schwärzt's noch gar, mir's immer doch nicht schwarz genug war», fügte Berliner noch hinzu: «Ersetzen Sie schwarz durch scharf, dann gilt das alles für Paulis Art und Weise, die Dinge anzusehen, mit denen er nicht ganz und gar übereinstimmt.» ${ }^{87}$

Pauli hatte sich bereits wegen dieser «pädagogischen Frage» an Heisenberg gewandt. Über die Arbeit selbst urteilte er mit schonungsloser Offenheit und erklärte, es sei bekanntlich jedes Mal eine Katastrophe, wenn Einstein sich öffentlich zur Quantenmechanik äußere. Um jedoch «durch die Publikation eine gewisse Gefahr einer Verwirrung der öffentlichen Meinung namentlich in Amerika - abzuwenden», legte er Heisenberg nahe, eine Erwiderung an das «Physical Review» zu senden.

Anschließend skizzierte Pauli die Form einer «pädagogischen Erwiderung» indem er eine Unterscheidung der Fälle von Systemen ohne Wechselwirkung und der Unabhängigkeit der Teilsysteme vorschlug. Zu diesem Zwecke sollte Heisenberg «Überlegungen über den Schnitt und die 
Möglichkeit seiner Verlegung an eine beliebige Stelle» einmal «nicht populär - mit Formeln - kurz darstellen», da die entsprechenden Ausführungen von von Neumann ihm «zu epsilontisch» seien. «Man muß verschiedene Schichten der Realität unterscheiden: Eine $R$, die alle Informationen enthält, die man durch Messungen an 1 und 2 erfahren kann, und eine (aus $R$ deduzierbare) $r$, die nur die Informationen enthält, die man durch Messungen an 1 allein erhalten kann. Man muß dann zeigen, wie die Bekanntgabe eines Meßresultates an 2 diskontinuierliche Änderungen von $r$ zur Folge hat. Und daß man notwendig auf Widersprüche geführt wird, wollte man versuchen, diese Änderungen ohne Bezugnahme auf 2 - etwa als «verborgene Eigenschaften> von 1 klassisch oder halbklassisch zu beschreiben.»

Abschließend erklärte Pauli, Heisenberg könnte bei dieser Gelegenheit «einmal in autoritativer Weise klarstellen, daß eine solche Ergänzung bei der Quantenmechanik nicht möglich ist, ohne ihren Inhalt abzuändern». ${ }^{88}$

Heisenberg hatte inzwischen Bohrs Erwiderung im Manuskript kennengelernt. Weil Bohr seine Gegenargumente mit Hilfe seines bekannten Doppelspaltexperiment durchführte, nahm Heisenberg Paulis Vorschlag an und verfaßte seinerseits einen Aufsatz mit dem Titel «Ist eine deterministische Ergänzung der Quantenmechanik möglich?» ${ }^{89}$

Schrödingers Aufsatz war inwzsichen zu «einer gemeinverständlich sein sollenden Darstellung des ganzen heute landläufigen Verfahrens ... mit kritischer Durchsetzung» angewachsen ${ }^{90}$. Vor einer Veröffentlichung wollte er gerne Paulis Ansicht darüber hören, ob er meine, daß der «Einsteinfall» restlos nichts zu denken gebe. «So meinten bisher alle, mit denen ich zum ersten Mal darüber sprach, weil sie ihr Kopenhagener Credo unum Sanctum gut gelernt hatten ... Aber klare Auskunft, warum alles so klar und einfach ist, bekam ich noch nicht.» ${ }^{91}$ Auch Pauli erklärte, der «Einsteinfalls enthalte in der Tat nichts «als sehr elementar-direkte Konsequenzen der Unbestimmtheitsrelationen»». ${ }^{92}$

Schrödinger schickte sein Manuskript dennoch Anfang August an die Redaktion der Naturwissenschaften. Arnold Berliner hatte inzwischen seine schon lange zu befürchtende Kündigung erhalten. Daß Schrödingers Aufsatz noch zur Publikation gelangte, ist sicherlich dem Einfluß Max von Laues zuzuschreiben, der in diesen düsteren Jahren der nationalsozialistischen Herrschaft mit größter Umsicht die Belange der Physik wirksam zu vertreten wußte.

Aber mit Berliners Ausscheiden kam eine wirkliche Aussprache zwischen den beiden Parteien nicht mehr zustande. Heisenbergs Stellungnahme 
konnte nicht mehr in den Naturwissenschaften aufgenommen werden, und die ganze Diskussion kam vorerst zum Stillstand.

Schrödingers Besorgnis, «Einstein, von Laue und er selbst habe sich auf diese Art vor der Nachwelt blamiert», konnte Max von Laue zunächst nur durch den Hinweis beschwichtigen, daß «jeder von uns dreien sich damit trösten muß, daß er in ganz guter Gesellschaft ist». ${ }^{93}$

\section{Literaturverzeichnis}

K. Baumann und R.Sexl [1984]: Die Deutungen der Quantentheorie. Braunschweig/Wiesbaden 1984 .

F. Bloch (1976): Heisenberg and the early days of quantum mechanics. Physics Today/December 1976. S.23-27.

M. Born (1927): Quantenmechanik und Statistik. Naturwiss. 15, 238-242 (1927).

M.Born (1928): Über die Bedeutung der Stoßvorgänge für das Verständnis der Quantenmechanik. Atti del congresso Internazionale dei Fisici. Como, 11.-20. Settembre 1927. V. Band 2. Bologna 1928. Dort S. 443-447; 589-591.

M. Born (1928/29): Über den Sinn der physikalischen Theorien. Göttinger Nachr., Geschäftl. Mitteilungen 1928/29, S.51-70.

M. Born (1955): Albert Einstein und das Lichtquantum. Naturwiss. 41, 425-431 (1955).

M. Born (1961): Bemerkungen zur statistischen Deutung der Quantenmechanik. In: Werner Heisenberg und die Physik unserer Zeit. Braunschweig 1961.

R. Carnap (1923): Über die Aufgabe der Physik. Kantstudien 28, 90-107 (1923).

M. Eckert und W.Pricha (1984): Die ersten Briefe Albert Einsteins an Arnold Sommerfeld. Phys. Bl. 40, 29-34 (1984).

A. Einstein (1921): Über ein den Elementarprozeß der Lichtemission betreffendes Experiment. SB der Preuß. Akad. Wiss. 1921, S.882-883.

L. Flamm (1927): Die neue Mechanik. Naturwiss. 15, 569-578 (1927).

V. Fock (1926): Zur Schrödingerschen Wellenmechanik. Z. Phys. 38, 242-250 (1926).

W.A. Fock (1962): «Über die Interpretation der QM.» In: Philosophische Probleme der modernen Naturwissenschaft. Allunionskonferenz Moskau 1958. Berlin 1962. Dort S. 189-212.

G. Gamow (1940): Basic principles of the new mechanics. Scient. monthly 51, 358-364 (1940).

O. Halpern und H. Thirring (1929): Die Grundgedanken der neueren Quantentheorie. Zweiter Teil. Erg. exakt. Naturw. 8, 367-508 (1929).

D. Hilbert, J.v. Neumann und L. Nordheim (1927): Über die Grundlagen der Quantenmechanik. Math. Annalen 98, 1-30 (1927).

H. Hönl und C. Eckart (1930): Grundzüge und Ergebnisse der Wellenmechanik. Physik. Z.31, 89-119, 145-165 (1930).

M. Jammer (1966): The conceptual Development of Quantum Mechanics. New York 1966.

P. Jordan (1927): Besprechung der 1. Auflage von Schrödingers Abhandlungen zur Wellenmechanik. Naturwiss. 15, 412-413 (1927). 
P. Jordan (1929): Besprechung der 2., vermehrten Auflage von Schrödingers Abhandlungen zur Wellenmechanik. Naturwiss. 17, 164-166 (1929).

H. Konno (1983): Slater's Evidence for the Genesis of the Bohr-Kramers-Slater Theory. Hist. Scientiarium 25, 39-52 (1983).

H. Konno (1978): The historical roots of Born's probabilistic interpretation. Jap. Stud. Hist. Sci. 17, 129-145 (1978).

A. Landé (1926): Neue Wege der Quantentheorie. Naturwiss. 14, 455-458 (1926).

A. Landé (1927): Spontane Quantenübergänge. Z. Phys. 42, 835-839 (1927).

A. Landé (1938): Critical remarks on the interpretation of quantum theory. J. Franklin Inst. 226, 83-98 (1938).

M.v. Laue (932): Zu den Erörterungen über Kausalität. Naturwiss. 20, 915-916 (1932).

M.v. Laue (1934 a): Statistische Physik. Handwörterbuch der Naturwissenschaften. Band 9, S. 537-550. Jena 1934.

M.v. Laue (1934b): Über Heisenbergs Ungenauigkeitsbeziehungen und ihre erkenntnistheoretische Bedeutung. Naturwiss. 32, 439-441 (1934).

M.v. Laue (1934c): Materie und Raum in der neuen Physik. Vossische Zeitung No.21 vom 25. Januar 1934.

M.v. Laue (1951): Materiewellen. Naturwiss. 38, 55-61 (1951).

E. Madelung (1926): Eine anschauliche Deutung der Gleichung von Schrödinger. Naturwiss. 14, 1004. (1926).

E. Madelung: Quantentheorie in hydrodynamischer Form. Z. Phys. 40, 322-326 (1926).

H.Margenau (1951): Begriffliche Grundlagen der Quantentheorie. Phys. Bl. 7, 342-354 (1951).

K.v. Meyenn (1984): Gespensterfelder und Materiewellen: Schrödingers Hang zur Anschaulichkeit. Phys. Bl. 40, 89-94. (1984).

A.Pais (1982): Max Born's statistical interpretation of quantum mechanics. Science 218, 1193-1198 (1982).

W. Pauli (1926): Quantentheorie. Handbuch der Physik, herausgegeben von H.Geiger und K. Scheel. Band 23, S.1-278. Berlin 1926.

W. Pauli (1927): Über Gasentartung und Paramagnetismus. Z. Phys. 41, 81-102 (1927).

W.Pauli (1929): Allgemeine Grundlagen der Quantentheorie des Atombaues. Müller-Pouillets Lehrbuch, Band 2, Teil 2, S. 1709-1842. Braunschweig ${ }^{11} 1929$.

W.Pauli (1933): Einige die Quantenmechanik betreffende Erkundigungsfragen. Z. Phys. 80, 573-586 (1933).

W.Pauli (1985): Wissenschaftlicher Briefwechsel mit Bohr, Einstein, Heisenberg u.a. Band II: 1930-1939. Berlin/Heidelberg/New York/Tokyo 1985.

M.Planck (1929): Erwiderung. Sitzungsber. Preuß. Akad. Wiss., phys.-math. Kl. (1929), S. CII-CIV.

E.A. Ruark (1935): Is the quantum mechanical description of physical reality complete? Phys. Rev. 48, 466-467 (1935).

M.Schottky (1921): Das Kausalproblem der Quantentheorie als eine Grundfrage der modernen Naturforschung überhaupt. Naturwiss. 9, 492-496, 506-511 (1921).

E.Schrödinger (1919): Über die Kohärenz in weitgeöffneten Bündeln. Ann.Phys. (4) 61, 69-86 (1919) 
E. Schrödinger (1926a): Quantisierung als Eigenwertproblem (Erste Mitteilung). Ann. Phys. (4) 70,361-376 (1926).

E.Schrödinger (1926b): Quantisierung als Eigenwertproblem (Zweite Mitteilung). Ann. Phys. (4) 79, 489-527 (1926).

E. Schrödinger (1926c): Quantisierung als Eigenwertproblem (Dritte Mitteilung). Ann. Phys. (4) $80,437-490$ (1926).

E. Schrödinger (1926d): Quantisierung als Eigenwertproblem (Vierte Mitteilung). Ann.Phys. (4) $81,109-139$ (1926).

E.Schrödinger (1928): Neue Wege der Physik. Elektrische Nachrichtentechnik 5, 485-488 (1928).

E. Schrödinger (1929a): Antrittsrede. Sitzungsber. Preuß.Akad. Wiss., phys.-math. Kl. (1929), S.C-CII.

E. Schrödinger (1929 b): Das Gesetz der Zufälle. Die Koralle 5, 417-418 (1929).

E. Schrödinger (1932): Indeterminismus in der Physik. Leipzig 1932.

E. Schrödinger (1934): Über die Unanwendbarkeit der Geometrie im Kleinen. Naturwiss. 22, 518-520 (1934).

E. Schrödinger (1935): Die gegenwärtige Situation in der Quantenmechanik. Naturwiss. 23, 807-812, 823-828, 844-849 (1935).

A. Sommerfeld (1919/1921): Atombau und Spektrallinien. Braunschweig 1919, ${ }^{2} 1921$.

A. Sommerfeld (1929): Einige grundsätzliche Bemerkungen zur Wellenmechanik. Physik. Z.30, 866-871 (1929).

H.Thirring (1928): Die Grundlagen der neueren Quantentheorie. Erster Teil. Erg. exakt. Naturwiss. 7, 384-431 (1928).

D.S. Villars (1929): What Happens During the Electron Jump? Nature 123, 240 (1929).

\section{Anmerkungen}

${ }^{1}$ Die große Zurückhaltung, mit der man damals der Lichtquantenhypothese begegnete, äußerte sich auch in Einsteins eigenen Zweifeln. Als er Max von Laue davon berichtete, war dieser sehr erfreut: «Ich habe, wie Sie wissen, nie viel für sie übrig gehabt.» (Vgl. M.v. Laues Schreiben an A.Einstein, 27. Dezember 1907.)

${ }^{2}$ Einstein an Sommerfeld, Juli 1910. Wiedergegeben in Eckert und Pricha (1984).

${ }^{3}$ Vgl.z. B. Einsteins Bemerkungen in einem Schreiben an H. A. Lorentz vom 23. November 1911, in dem er auf ein Problem der Rotationsbewegung eines Dipols in einem Strahlungsfelde zurückkam, das während des ersten Solvay-Kongresses im Oktober diskutiert worden war.

${ }^{4}$ Einen möglichen Ausweg erblickte Wolfgang Pauli anfangs noch in der kleinen Unschärfe der Quantenzustände infolge der Übergangsprozesse, die zu der Annahme berechtigten, nicht alle angeregten Atome als vollkommen gleich zu betrachten. (Vgl. Pauli [1926], S.11.)

${ }^{5}$ Nach einem Schreiben von Einstein an Ehrenfest, 15. März 1921.

${ }^{6}$ Schrödinger (1919).

${ }^{7}$ Sommerfeld (1919), S. VIf.

${ }^{8}$ Sommerfeld (1919), S. 379.

${ }^{9}$ Sommerfeld (1921), S. 475. 
${ }^{10}$ Aus einem Brief von Einstein an Ehrenfest, 18. September 1925.

${ }^{11}$ So werden die Führungsfelder beispielsweise in einem Schreiben Einsteins vom 23. März 1922 an Ehrenfest erwähnt.

${ }^{12}$ Schottky (1921), S. 509.

${ }^{13}$ Aus einem Brief von Ehrenfest an Einstein, 17. Januar 1922.

${ }^{14}$ Vgl.hierzu Bohrs Brief vom 9. Januar 1924 an Rutherford, in dem er die Wellentheorie des Lichtes als eine Art Glaubensbekenntnis bezeichnete.

${ }^{15}$ Siehe hierzu Konno (1983).

${ }^{16}$ Aus einem Brief von Schrödinger an Bohr, 24. Mai 1924.

17 Aus einem Brief Schrödingers an Reichenbach, 25. Januar 1924.

${ }^{18}$ Siehe hierzu die Briefe von Heisenberg an Pauli, 30. September 1924, und an Sommerfeld, 18. November 1924.

19 Aus einem Brief von Pauli an Bohr, 2. Oktober 1924.

${ }^{20}$ Siehe Paulis Schreiben vom 2. Oktober 1924 an Bohr.

${ }^{21}$ Aus einem Brief von Einstein an Born, 29. April 1924. Wie Einstein in seinen Briefen (vom 31. Mai und 12. Juli 1924) an Ehrenfest schrieb, hatte er auch in einem Berliner Kolloquiumsvortrag zur Bohr-Kramers-Slater-Theorie Stellung genommen.

${ }^{22}$ Siehe den Brief von Pauli an Bohr, 12. Dezember 1924. Vgl. auch die ähnliche Auffassung, die Ehrenfest in seinem Brief vom 9. Januar 1925 an Einstein vertrat.

${ }^{23}$ Vgl. hierzu Paulis Schreiben vom 15. Dezember 1924 an Landé.

${ }^{24}$ Nach einem Brief von Einstein an Lorentz, 16. Dezember 1924.

${ }^{25}$ Nach einem Brief von Ehrenfest an Einstein, 9. Januar 1925.

${ }^{26}$ Pauli (1926), S. 85.

${ }^{27}$ Aus einem Schreiben von Kronig an Goudsmit, 5. Juni 1925.

${ }^{28}$ Aus einem Brief an Franck, 30. März 1925.

${ }^{29}$ Aus einem Brief von Pauli an Kramers, 27. Juli 1925.

${ }^{30}$ Siehe hierzu Halpern und Thirring (1929).

${ }^{31}$ Siehe beispielsweise Einsteins Brief an Ehrenfest, 28. August 1926.

${ }^{32}$ In einem Schreiben an Ehrenfest vom 20. September 1925.

${ }^{33}$ Nach einem Brief von Einstein an Ehrenfest, 12. April 1926.

${ }^{34}$ Aus Einsteins Brief an Ehrenfest vom 28. August 1926.

${ }^{35}$ Aus Einsteins Brief an Ehrenfest vom 23. August 1926.

${ }^{36}$ Vgl. hierzu Einsteins Schreiben vom 16. Dezember 1924 an Lorentz.

37 Aus einem Schreiben von Born an Einstein, 15. Juli 1925.

${ }^{38}$ Aus einem Brief von Schrödinger an Planck, 26. Februar 1926.

${ }^{39} \mathrm{Vgl}$. Schrödinger (1926b), S. 514.

${ }^{40}$ Vgl. die Briefe von Wien an Schrödinger, 13. Mai 1926; und von Einstein an Ehrenfest, 12. April 1926.

${ }^{41}$ Siehe Paulis Brief vom 12. April 1926 an Jordan.

${ }^{42}$ Danach sollte die Norm der Wellenfunktion $\Psi$ die räumliche Ladungsdichte der Elektrizität darstellen. Vgl. Schrödinger (1926d) S. 134 ff.

${ }^{43}$ Selbst im entfernten Amerika war man sogleich darauf aufmerksam geworden. "What do you think of Schrödinger's new mechanics?», schrieb G.Breit am 2. Juni 1926 aus Washington. «Laporte and I are very much impressed.»

${ }^{44}$ Die Einladungen nach Berlin und München waren schon im April an Schrödinger ergangen, 
wie aus seinem Schreiben vom 28. April 1926 an Sommerfeld zu ersehen ist: «Ich bin auch nach Berlin aufgefordert worden, zu irgendeiner Gauvereinssitzung des Sommersemesters zu kommen. Ich wage auch da keine definitive Zusage ... Vielleicht könnt' ich es dann so machen, ... daß ich auf der Rückreise über München komme.» Schrödingers Vorträge in Berlin wurden zum 16. und 17. Juli angesetzt.

${ }^{45}$ Einsteins Brief vom 28. August an Ehrenfest.

${ }^{46}$ Aus v. Laues Brief an Schrödinger, 12. Oktober 1926.

${ }^{47}$ Einsteins Brief vom 28. August an Ehrenfest.

${ }^{48}$ Brief von Heisenberg an Pauli, 28. Juli 1926.

${ }^{49}$ Vgl. hierzu Sommerfelds Schreiben vom 26. Juli 1926 an Pauli.

${ }^{50}$ Brief von Schrödinger an Wien, 25. August 1926.

${ }^{51}$ Vgl. hierzu Schrödingers Briefwechsel mit Born und Jordan aus dem Jahre 1927 und Jordans Habilitationsvortrag über «Kausalität und Statistik in der modernen Physik», der Anfang Februar 1927 in den «Naturwissenschaften» erschienen war.

52 Vgl. hierzu Born (1927), S.241, und Born (1928), S. 443.

${ }^{53}$ Siehe Pauli (1929), S.1835f.; und Kramers Diskussionsbemerkung zu Bohrs Vortrag auf dem Como-Kongreß im September 1927 (Band 2 der Kongreßberichte, S. 591 f.).

${ }^{54}$ Siehe Schrödingers Brief vom 17. November 1926 an Joos und vom 19. November an Kramers.

${ }^{55}$ Vgl. Jordan (1927), S. 413.

${ }^{56}$ Brief von Born an Schrödinger, 16. Mai 1927.

${ }^{57}$ Siehe den Brief von Heisenberg an Jordan vom 24. November 1926.

${ }^{58}$ Brief von Heisenberg an Pauli, 31. Mai 1927.

${ }^{59}$ Diese Bezeichnungsweisen werden in den Briefen von London an Schrödinger, 19. August 1926; von Jordan an Schrödinger, Mai 1927; von Schrödinger an Lorentz, 23. Juni 1927, und von Heisenberg an Born, Jordan und Smekal vom 29. Oktober 1926 verwendet.

${ }^{60}$ Siehe Paulis Schreiben vom 22. November 1926 an Schrödinger.

${ }^{61}$ Brief von Heisenberg an Sommerfeld, 6. November 1926. Siehe auch Paulis Schreiben an Wentzel und Pauli (1927), S. 83; Jordan (1927), S. 108, und Born (1927), S. 242.

${ }^{62}$ Brief von Heisenberg an Jordan, 7. März 1927.

${ }^{63}$ Vgl. hierzu Jordan (1929), S. 165.

${ }^{64}$ Nach einem Brief von Einstein an Weyl vom 26. April 1927.

${ }^{65}$ Aus Borns Brief vom 6. November 1960 an Schrödinger.

${ }^{66}$ Heisenberg in seinen Anmerkungen zu Bohrs Vortrag auf der Volta-Feier (Kongreßberichte, Band 2, S.593f.).

${ }^{67}$ Siehe Born (1927), S. 239.

${ }^{68}$ Vgl. Born (1928/29), S. 68.

${ }^{69}$ Brief von Sommerfeld an Wentzel, 19. Dezember 1926. Siehe hierzu auch v. Meyenn (1984), S. 94.

${ }^{70}$ Vgl. Planck (1929), S. CII.

${ }^{71}$ Aus einem Brief von Einstein an Weyl, 26. April 1927. Dennoch gehörte Einstein mit zu den ersten, die Heisenberg und Schrödinger für den Nobelpreis vorschlugen. (Vgl. Einsteins Schreiben vom 21. Januar 1928 an Ehrenfest.)

72 Siehe Bohrs Schreiben vom 5. November 1928 an Oseen.

${ }^{73}$ Schrödinger (1928), S.487f. 
${ }^{74}$ Schrödinger (1934), S. 519.

${ }^{75}$ Schrödinger (1929b), S.418.

${ }^{76}$ Brief von Schrödinger an Weyl, 6. November 929.

${ }^{77}$ Ehrenfest in einem Schreiben an Kramers, 5. September 1928.

${ }^{78}$ Vgl. Schrödingers Brief vom 5. Mai 1927 an Ehrenfest.

${ }^{79}$ Brief von Ehrenfest an Schrödinger, 28. September 1932.

${ }^{80}$ In humoristische Form gekleidet, hat Ehrenfest einen Auszug davon für eine Festnummer der «Göttinger Nachrichten» zu Max Borns 50. Geburtstag zusammengestellt. (Siehe Pauli [1985], S. 124.)

${ }^{81}$ Brief von Schrödinger an Ehrenfest, 30. September 1932.

${ }^{82}$ In diesem Sinne äußerte sich Wilhelm Wien in einem Schreiben vom 6. September 1927 an Schrödinger: «Gegen die Auffassung de Broglies und Borns habe ich nichts einzuwenden, wenn man sie als vorläufige Darstellungsweise betrachtet. ... Aber ich bin vollkommen davon überzeugt, daß man sich niemals damit zufrieden geben wird, daß in den elementaren Vorgängen (Wahrscheinlichkeiten〉 vorkommen, also die Kausalität ausgeschaltet wird.»

83 v. Laue (1932), S. 915.

${ }^{84}$ Aus v. Laues Schreiben vom 22. August 1934 an Einstein. Auch v. Laues Skepsis gegen die Kopenhagener Deutung hat sich in den späteren Jahren nicht gewandelt. (Vgl.z. B. v. Laue [1951], S.60.)

${ }^{85}$ Aus einem Schreiben von Gehrcke und Stark an Planck, 27. Mai 1934.

${ }^{86}$ Berliner, der früher eher unter der Fülle der ihm zur Veröffentlichung angebotenen Manuskripte zu leiden hatte, mußte jetzt häufig um Beiträge bitten, um seine Spalten zu füllen. (Vgl.beispielsweise Berliners Korrespondenz mit Sommerfeld u. a.)

${ }^{87}$ Brief von Berliner an Schrödinger, 1. Juli 1935.

${ }^{88}$ Brief von Pauli an Heisenberg, 15. Juni 1935.

${ }^{89}$ Dieser Aufsatz ist in Pauli (1985), S.409-418, enthalten.

${ }^{90}$ Brief von Schrödinger an von Laue, 25. Juli 1935.

${ }^{91}$ Brief von Schrödinger an Pauli, Juli 1935.

${ }^{92}$ Brief von Pauli an Schrödinger, 9. Juli 1935.

${ }_{93}$ Brief von Laues an Schrödinger, 11. März 1936.

\section{Summary}

The most antagonistic aspect of the old quantum theory became apparent in the wave-particle dualism of the radiation phenomena. In spite of the efforts made by the defenders of the extreme quantum theoretical point of view, who believed in the possibility of a full representation of those phenomena in the particle picture, most physicists inclined toward a wave-like description. Only after the discovery of the Compton-effect in 1923 the impossibility of such a unitary position was generally recognized.

The first conceptual synthesis of these opposite views emerged after the discovery of the new Quantum-and-Wave-Mechanics in the so called Copenhagen Interpretation. Wolfgang Pauli was one of the most fervent promotors of these new ideas. Schrödinger on the contrary became one of the strongest opponents, seeking for a formulation of the new theories with the help of concepts of our daily experience and without the hatred "quantum jumps". A general 
controversy about these problems by the most distinguished exponents of different viewslike Einstein, Bohr, Schrödinger, Pauli, and Heisenberg-was aspired in the thirties by the editor of «Die Naturwissenschaften», Arnold Berliner. Only the political events in Germany of that time prevented a full realization of such a public discussion. So the general debate of these fundamental issues became delayed until after the war.

Dr. Karl von Meyenn

Seminari d'Historia de les Ciènces

Facultat des Ciènces

Universitat Autonoma De Barcelona

Bellaterra (Barcelona)

España 
